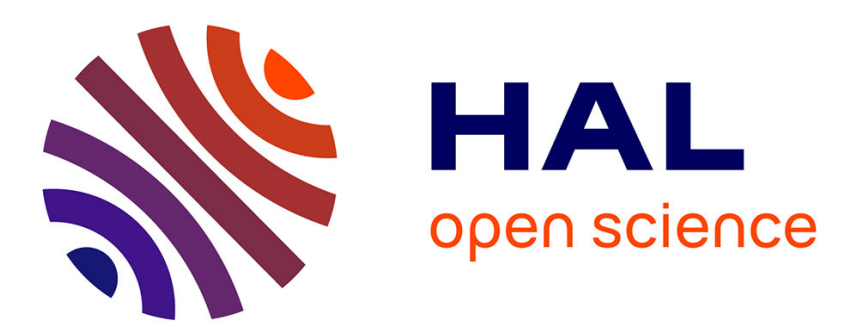

\title{
Effect of Bias on the Response of GaN Axial p-n Junction Single-Nanowire Photodetectors
}

Sergi Cuesta, M. Spies, V. Boureau, F. Donatini, Mö̈ra Hocevar, M. den Hertog, E. Monroy

\section{To cite this version:}

Sergi Cuesta, M. Spies, V. Boureau, F. Donatini, Moïra Hocevar, et al.. Effect of Bias on the Response of GaN Axial p-n Junction Single-Nanowire Photodetectors. Nano Letters, 2019, 19 (8), pp.5506-5514. 10.1021/acs.nanolett.9b02040 . hal-02326742

\section{HAL Id: hal-02326742 \\ https://hal.science/hal-02326742}

Submitted on 7 Jun 2021

HAL is a multi-disciplinary open access archive for the deposit and dissemination of scientific research documents, whether they are published or not. The documents may come from teaching and research institutions in France or abroad, or from public or private research centers.
L'archive ouverte pluridisciplinaire HAL, est destinée au dépôt et à la diffusion de documents scientifiques de niveau recherche, publiés ou non, émanant des établissements d'enseignement et de recherche français ou étrangers, des laboratoires publics ou privés. 
This document is the unedited Author's version post review of a Submitted Work that was subsequently accepted for publication in Nano Letters, copyright (C) American Chemical Society after peer review. To access the final edited and published work see [https://pubs.acs.org/doi/full/10.1021/acs.nanolett.9b02040].

\title{
Effect of bias on the response of GaN axial p-n junction single-nanowire photodetectors
}

\author{
S. Cuesta ${ }^{\dagger}$, M. Spies ${ }^{\dagger}$, V. Boureau ${ }^{\dagger}$, F. Donatini ${ }^{\dagger}$, M. Hocevar ${ }^{\dagger}$, M. I. den Hertog ${ }^{\dagger}$, \\ and E. Monroy ${ }^{*}, \ddagger$
}

${ }^{+}$Univ. Grenoble-Alpes, CNRS-Institut Néel, 25 av. des Martyrs, 38000 Grenoble, France

‡ Univ. Grenoble-Alpes, CEA-IRIG-PHELIQS, 17 av. des Martyrs, 38000 Grenoble, France

OrcIDs:

Sergi Cuesta : 0000-0003-0262-5875

Maria Spies: 0000-0002-3570-3422

Victor Boureau: 0000-0001-6251-5892

Fabrice Donatini 0000-0001-6556-1683

Moïra Hocevar : 0000-0001-5949-5480

Martien I. den Hertog: 0000-0003-0781-9249

Eva Monroy: 0000-0001-5481-3267

*E-mail: eva.monroy@cea.fr

\begin{abstract}
We present a comprehensive study of the performance of GaN single-nanowire photodetectors containing an axial $p-n$ junction. The electrical contact to the $\mathrm{p}$ region of the diode is made by including a $\mathrm{p}^{+} / \mathrm{n}^{+}$tunnel junction as cap structure, which allows the use of the same metal scheme to contact both ends of the nanowire. Single-nanowire devices present the rectifying current-voltage characteristic of a $p-n$ diode, but their photovoltaic response to ultraviolet radiation scales sublinearly with the incident optical power. This behavior is attributed to the dominant role of surface states. Nevertheless, when the junction is reverse biased, the role of the surface becomes negligible in comparison to the drift of photogenerated carriers in the depletion region. Therefore, the responsivity increases by about three orders of magnitude and the photocurrent scales linearly with the excitation. These reverse-biased nanowires display decay times in the range of $\approx 10 \mu \mathrm{s}$, limited by the resistance $\times$ capacitance time constant of the setup. Their ultraviolet/visible contrast of several orders of magnitude is suitable for applications requiring high spectral selectivity. When the junction is forward biased, the device behaves as a GaN photoconductor, with an increase of the responsivity at the price
\end{abstract}


of a degradation of the time response. The presence of leakage current in some of the wires can be modeled as a shunt resistance which reacts to the radiation as a photoconductor and can dominate the response of the wire even under reverse bias.

KEYWORDS: Photodetector, nanowire, ultraviolet, GaN, axial junction

\section{INTRODUCTION}

The required transition from an energy network based on fossil fuels towards a larger contribution of renewable resources requires, among other issues, the generalized implantation of solar cells. With this target in mind, huge research efforts are oriented towards increasing the solar cell power conversion efficiency, as a way to decrease the total system cost in dollar per Watt. In this context, nanowires are attracting a lot of interest ${ }^{1-3}$ thanks to their high tolerance to lattice mismatch, which enables the implementation of multi-junction solar cells with more flexibility than planar structures. Furthermore, the light concentration capabilities of nanowires make it possible to reduce the amount of active material without degradation of the total absorption. ${ }^{4,5}$

In the domain of ultraviolet, visible and infrared photodetectors, the nanowire geometry constitutes an exciting approach to get around the speed-responsivity trade-off. The low electrical cross-section of nanowires results in low electrical capacitance, while maintaining the same total absorbance as planar layers. ${ }^{4-6}$ Another interesting feature is the compatibility with silicon technology, which opens the possibility of monolithic integration of detector and readout. The simplicity of their transfer into flexible materials ${ }^{7,8}$ is also particularly attractive, and opens perspectives for the development of wearable devices.

Various designs have been considered as nanowire photodetectors ${ }^{9-11}$ or solar cells. ${ }^{12-14}$ In the case of photodetectors, most works focus on the metal-semiconductor-metal architecture, with either ohmic or Schottky contacts. Such devices present high gain, but they are strongly sublinear and their time response is generally in the millisecond range. Reduction of the dark current and enhancement of the responsivity are possible by generating an internal electric field in the structure, e.g. by inserting a heterostructure of materials with spontaneous polarization. ${ }^{15}$ Also, the linearity can be recovered if the nanowires are thin enough to be fully depleted due to the effect of surface states. ${ }^{16-18}$ However, in all these cases, the response time remains long (millisecond range) due to the important role of surface states on the transport process. 
When the target is a quantitative measurement of the incident radiation, $\mathrm{p}-\mathrm{n}$ photodiodes are generally preferred over metal-semiconductor-metal architectures because of their lower dark current and the linearity of their response. Furthermore, photodiodes can be operated at zero-bias (self-powered devices), although speed and responsivity are enhanced under reverse bias. Within one nanowire, the arrangement of the $\mathrm{p}$ and $\mathrm{n}$ regions can be radial (core-shell junction, e.g. $\mathrm{Si}^{19}{ }^{19} \mathrm{GaAs},{ }^{5,20,21} \mathrm{GaAsP},{ }^{22,23}$ or $\mathrm{GaN}^{8,24}$ ) or axial (e.g. $\mathrm{Si}^{25} \mathrm{GaAs}^{26}$ or $\mathrm{GaN}^{27-29}$ ). In the particular case of GaN p-n junctions, Zhang et al. ${ }^{24}$ reported a single-nanowire core-shell junction with a peak UV responsivity of $0.14 \mathrm{~A} / \mathrm{W}$ at zero bias and $0.157 \mathrm{~A} / \mathrm{W}$ at $-1 \mathrm{~V}$ bias. Unfortunately, there was no information on the linearity of the devices or on the optical area that was considered for the calculation of the responsivity. On the other hand, de Luna Bugallo et al. $^{28}$ demonstrated a photodiode fabricated by planarization (using spin-on-glass), mesa etching and contacting of an ensemble of $\mathrm{GaN}$ nanowires containing an axial $p-n$ junction. The detector active area was $300 \times 300 \mu \mathrm{m}^{2}$. In this latter case, the device presented a peak responsivity of $0.47 \mathrm{~A} / \mathrm{W}$ at $-1 \mathrm{~V}(0.09 \mathrm{~A} / \mathrm{W}$ at zero bias $)$. A responsivity of $0.47 \mathrm{~A} / \mathrm{W}$ at $356 \mathrm{~nm}$ implies an external quantum efficiency higher than unity (to be precise, 1.6), i.e. the device presented photocurrent gain under bias voltages that were not compatible with impactionization processes. This abnormal responsivity was later attributed to photoconductive gain associated with the behavior of surface states under illumination, observed under both forward and reverse bias. ${ }^{29}$ At zero bias, the responsivity is linear with the optical power for irradiance below $600 \mu \mathrm{W} / \mathrm{cm}^{2}$, above that value it saturates. This is in contrast with $\mathrm{Si}$ and GaAs single nanowires containing p-n junctions, which have demonstrated linear behavior at zero bias for power densities higher than $1 \mathrm{~W} / \mathrm{cm}^{2} .{ }^{19,21,25,26}$ The early saturation of the photoresponse might also be associated with surface phenomena. Finally, in both reports on GaN nanowires (refs. ${ }^{24}$ and ${ }^{28}$ ), the devices presented non-exponential photocurrent transients with response times in the tens-of-milliseconds range under reverse bias, slowing down under forward bias. These response times are too slow to be limited by the resistance $\times$ capacitance product of the devices, and the slowness and non-exponential behavior could be explained by surface trapping effects. As a whole, these characteristics show a clear deviation from the behavior of a planar $p-n$ junction, which rises questions on the feasibility of a nanowire photodetector that is not dominated by surface phenomena. There is hence a motivation to explore the behavior of a single nanowire, i.e. with less perturbation by the device fabrication processes and without the dispersion of properties introduced by the nanowire ensemble. 
Nonlinear gain associated with impact-ionization phenomena (avalanche amplification) can appear when the devices are strongly reverse biased. Nanowire avalanche photodiodes have been demonstrated in InP-based ${ }^{30,31}$ and GaAs-based ${ }^{32-34}$ junctions. These devices, operated under high reverse bias, are interesting for application in single-photon counting.

For an application as solar cells, theoretically, radial junctions appear as advantageous with respect to axial junctions due to the short carrier collection length (determined by the nanowire diameter) combined with a large absorbing volume (determined by the nanowire length, in general longer than the absorption depth). ${ }^{35}$ However, in comparison with axial structures, the core-shell geometry complicates the implementation of multi-junctions. Looking at the experimental results, core-shell p-n junctions show relatively low open-circuit voltage, which points to a lower junction quality in comparison with axial junctions.

In view of the state of the art, it is important to get a deep understanding of the operation mechanisms of a single-nanowire photodetector containing an axial $\mathrm{p}-\mathrm{n}$ junction, as most reports refer to nanowire ensemble. By assessing the behavior of an individual nanowire, we can identify intrinsic mechanisms without the noise introduced by the dispersion of size or doping density in the nanowire ensemble. Characterization must go beyond direct exposure to the global standard solar spectrum and analyze the device performance as a function of the incident wavelength and optical power. The correlation between time response, responsivity, linearity and bias will allow us to understand the physical processes that are at the origin of slow transients and photocurrent gain.

With this purpose, this paper presents an in-depth analysis of single-nanowire GaN axial p-n junctions. Compared to other materials, $\mathrm{GaN}$ is mechanically, thermally and electrically robust. Moreover, almost defect-free wurtzite GaN nanowires can be obtained by plasmaassisted molecular-beam epitaxy (PAMBE) ${ }^{36,37}$ In this work, single nanowires were characterized in terms of responsivity, spectral selectivity and switching speed. Finally, we considered the variation of the response with the optical power and with bias.

\section{SAMPLE DESCRIPTION AND EXPERIMENTAL METHODS}

Self-assembled $(000 \overline{1})$-oriented ${ }^{38} \mathrm{GaN}$ nanowires were synthesized on $n$-Si(111) substrates using PAMBE, at a substrate temperature $T_{\mathrm{S}}=810^{\circ} \mathrm{C}$, and with a growth rate of $330 \mathrm{~nm} / \mathrm{h}$. The growth started with the deposition of an AlN buffer using a two-step procedure, as described elsewhere. ${ }^{39}$ Then, a $1.5-\mu \mathrm{m}$-long $\mathrm{GaN}$ stem n-doped with $\mathrm{Si}$ at $[\mathrm{Si}]=1 \times 10^{18} \mathrm{~cm}^{-3}$ (value 
estimated from Hall effect measurements using the Van der Pauw method on planar Si-doped $\mathrm{GaN}$ layers) was grown under nitrogen-rich conditions $(\mathrm{Ga} / \mathrm{N}$ flux ratio $=0.25)$. This was followed by an 80-nm-long non-intentionally-doped region, and a 700-nm-long Mg-doped section $\left(\mathrm{Mg}\right.$ beam equivalent pressure $\left.=9.6 \times 10^{-8} \mathrm{Torr}\right)$. Finally, the nanowire was capped with $70 \mathrm{~nm}$ of Mg-doped GaN (Mg beam equivalent pressure $=1.5 \times 10^{-7}$ Torr), and $135 \mathrm{~nm}$ of Sidoped GaN, with a Si flux corresponding to $[\mathrm{Si}]=5 \times 10^{19} \mathrm{~cm}^{-3}$ in planar layers. This $\mathrm{p}^{+} / \mathrm{n}^{+}$cap structure was thought to enable the use of the same metal scheme to contact both ends of the nanowire, since they are both n-type doped. The band profile along the nanowires was calculated using the Nextnano software ${ }^{40}$ following a one-dimensional approximation, with the result depicted in figure 1 (a). At the $\mathrm{p}^{+} / \mathrm{n}^{+}$tunnel junction (indicated by a red circle) electrons can tunnel from the conduction band of $\mathrm{n}^{+}-\mathrm{GaN}$ to the valence band of $\mathrm{p}^{+}-\mathrm{GaN}$. Such a tunnel junction is currently used as a contact scheme for GaN-based planar LEDs, ${ }^{41,42}$ and it has also been validated in the case of GaN nanowire LEDs. ${ }^{43}$

To electrically contact individual specimens, the as-grown nanowires were sonicated in isopropanol, and the solution was dispersed on an n-type $\operatorname{Si}(100)$ wafer covered with $200 \mathrm{~nm}$ $\mathrm{SiO}_{2}$ and $40 \mathrm{~nm}$ of stoichiometric $\mathrm{Si}_{3} \mathrm{~N}_{4}$. Contact pads and marker structures were previously defined by optical lithography, electron beam evaporation of Ti/Au ( $5 \mathrm{~nm} / 35 \mathrm{~nm})$ and lift-off. Individual nanowires were selected and contacted using electron beam lithography and deposition of Ti/Al (10 nm/120 nm). Scanning electron microscopy (SEM) images of contacted nanowires were recorded in a field-emission SEM Zeiss Ultra 55 microscope operated at $3 \mathrm{kV}$ (see figures $1(b-c)$ ).

To evaluate the current-voltage (I-V) characteristics, the nanowires were directly connected to an Agilent 4155C semiconductor parameter analyzer and biased over a range of $\pm 10 \mathrm{~V}$. Note that the n-type nanowire base was grounded and positive bias was defined as positive voltage applied to the Mg-doped cap, as indicated in figures 1(b-c).

Kelvin probe force microscopy (KPFM) experiments were carried out using the peak-force frequency modulation technique in a Dimension Icon-PT atomic force microscope equipped with a PFQNE-AL probe from Bruker. The measurements were recorded in the air, scanning along the nanowire axis. To detect and prevent measurement artifacts, it was verified that the same result was obtained when scanning from the $\mathrm{p}$ to the $\mathrm{n}$ contact as well as the other way around.

Measurements of the photocurrent as a function of the optical power were made with 
illumination from an unfocused continuous-wave $\mathrm{HeCd}$ laser $(325 \mathrm{~nm})$ chopped at $30 \mathrm{~Hz}$. Single nanowires were directly connected to the $10^{6} \mathrm{~A} / \mathrm{V}$ transimpedance amplifier integrated in a lock-in amplifier (Stanford Research Systems SR830). To measure the spectral response, we used a similar configuration, but with the excitation from a $450 \mathrm{~W}$ xenon lamp passed through a grating monochromator (Gemini 180 Jobin Yvon).

Time response measurements were performed by connecting the nanowires in series with a power supply and a $10 \mathrm{k} \Omega$ load resistance. The voltage drop in the load resistance is monitored with a TDS2022C oscilloscope. To study photodiodes with very different time response, two measurement setups were used. For nanowires reacting in the microsecond range, the excitation source was a pulsed Nd-YAG laser $(\lambda=266 \mathrm{~nm}, 1$ ns pulses with a frequency of $8 \mathrm{kHz})$. For nanowires with a time response in the millisecond range, the same continuous-wave $\mathrm{HeCd}$ ultraviolet laser as in previous setups was used, chopped at $2 \mathrm{~Hz}$.

Electron beam-induced current (EBIC) imaging was carried out in a FEI Inspect F50 fieldemission SEM operated at $10 \mathrm{kV}$ with a beam current of $60 \mathrm{pA}$. The microscope is equipped with an in-house EBIC system. The current collected at the n-side contact of the nanowire was measured using a Keithley 6485 picoammeter.

\section{RESULTS}

Top-view SEM images of contacted single p-n junction GaN nanowires are shown in figures 1(b) and (c), corresponding to specimens labelled NW1 and NW2, respectively. The location of the $\mathrm{n}$ and $\mathrm{p}$ regions of the junction, and the polarization orientation for the electrical measurements are indicated in the figures. The surface potential of the sample was probed using KPFM to verify the presence of the junction between the contacts, as illustrated in figures 1 (df) for sample NW1. Figures 1(d) and (e) show its height and probe contact potential difference (CPD) maps, respectively. The CPD profile along the nanowire following the arrow in figure 1(e) is plotted in figure 1(f), where the contact regions are indicated as shadowed areas. A CPD increase is identified starting at $150 \mathrm{~nm}$ from the p-contact edge towards the $\mathrm{n}$-side of the nanowire, with a variation that is well described by a sigmoidal function. The location of the inflection point around $230 \pm 30 \mathrm{~nm}$ fits the expected location of the $\mathrm{p}-\mathrm{n}$ junction in the structure. The magnitude of the CPD variation does not correspond to the built-in potential of the junction due to the presence of surface states and the associated band bending. Yet, the measured value $(\triangle \mathrm{CPD}=410 \pm 80 \mathrm{mV})$ is comparable to measurements performed in $\mathrm{p}$ - $\mathrm{n}$ junctions contained in 
GaN nanowires with similar diameter. ${ }^{44,45}$ We observe that the CPD signal presents a clear increase in the vicinity of the p-contact. This indicates that the metal is partially depleting the p-doped region. This is explained by the fact that the metallization scheme was chosen to be an ohmic contact for the $\mathrm{n}$ and $\mathrm{n}^{+}$regions, but it extends towards the $\mathrm{p}$-region generating a potential barrier.

Figures $1(\mathrm{~g})$ and $(\mathrm{h})$ display the I-V characteristics in the dark of nanowires NW1 and NW2, respectively. Sample NW1 shows the rectifying behavior representative of a p-n junction. In contrast, sample NW2 presents asymmetric transport properties, but with significant leakage current under negative bias associated with an early breakdown at $-2.5 \mathrm{~V}$. The leakage in this wire might be related to the proximity of the junction to the p-contact, as described in the figure $\mathrm{S} 1$ of the Supporting Information.

The variation of the photocurrent $\left(I_{p h}\right)$ as a function of the incident optical power $(P)$ was studied under chopped $(30 \mathrm{~Hz})$ illumination at $325 \mathrm{~nm}$. The power dependence of the photocurrent measured at $-3,0$ and $+3 \mathrm{~V}$ bias is presented in figures 2 (a) and (b) for samples NW1 and NW2, respectively. The experimental results can be fitted with a power law $I_{p h} \sim P^{\beta}$, where $\beta$ is extracted from the slope of the apparent linear fits in the log-log plots (solid lines in the figures). Not that $\beta=1$ corresponds to a linear response of the device to the optical power, illustrated by a dashed grey line. Figures 2(c) and (d) represent both the variation of the parameter $\beta$ and the photocurrent under $0.13 \mathrm{~W} / \mathrm{cm}^{2}$ excitation as a function of bias, measured between $-5 \mathrm{~V}$ and $+5 \mathrm{~V}$.

If we concentrate first on the photovoltaic (zero-bias) response, figure 2 shows that both nanowires are strongly sublinear. A similar result was previously observed for GaN-based single-nanowire photodetectors where the internal electric field was originating from the polarization difference generated by the presence of a GaN/AIN heterostructure in the undoped region of an n-i-n nanowire. ${ }^{15}$ Even fully-depleted GaN/AlN heterostructured single-nanowire photodetectors, which behave linearly under bias, loose their linearity at zero-bias operation. ${ }^{18}$ This behavior is in contradiction with the performance of GaN photovoltaic devices based on planar layers, since both planar p-n junctions and Schottky diodes display $\beta=1$ at zero bias. ${ }^{46}$ Note that single $\mathrm{Si}$ and GaAs p-n junction nanowires display also a linear behavior at zero bias. ${ }^{19,21,25,26}$ Therefore, the origin of the observed nonlinearity might be associated to the high sensitivity of the GaN nanowires to surface states or to the nonlinear behavior of the potential barrier at the p-contact. 
Let us focus now on the response under bias. In the case of sample NW1 displaying low leakage current, the photocurrent scales linearly with the optical power for $\mathrm{V}_{\mathrm{B}}<0$, without any trace of saturation for incident power densities as high as $500 \mathrm{~mW} / \mathrm{cm}^{2}$ (see figure 2(a)). This power density is three orders of magnitude higher than in previous reports on $\mathrm{GaN}$ nanowires containing axial p-n junctions. ${ }^{28}$ For $\mathrm{V}_{\mathrm{B}}>0, \beta$ decreases when increasing the voltage (see figure 2(c)). This is attributed to the absence of a depletion region under forward bias. In this regime, the photodiode adopts a resistor behavior, similar to a simple GaN photoconductor. Previous studies of single-nanowire photoconductors show a sublinear response to the optical power $(\beta<1),{ }^{15,47}$ unless the nanowire is fully depleted, ${ }^{18}$ similar to planar metal-semiconductormetal photodetectors. ${ }^{46}$ The linearity (sublinearity) of the nanowires under reverse (forward) bias has been verified in various specimens (see figure S2 of the Supporting Information).

In the case of NW2, the response is sublinear in the whole bias range under study $( \pm 5 \mathrm{~V})$, as depicted in figures 2(b) and (d). In figure 2(d), $\beta$ increases under reverse bias, but remains far from unity. We explain this behavior by the existence of a leakage current path that can be modeled as a shunt resistance. The presence of a shunt resistance is consistent with the I-V characteristics of the device. The resistance varies non-linearly with the incident optical power and can then dominate the nanowire photoresponse.

Additionally, a relevant parameter to evaluate a photodetector performance is the responsivity $\left(R_{p h}\right)$, which is the ratio between the detected photocurrent $\left(I_{p h}\right)$ and the incident optical power $(P)$ :

$$
R_{p h}=\frac{I_{p h}}{P}=\frac{I_{p h}}{\Phi A_{o p t}}
$$

where $\Phi$ is the optical power density impinging on the detector and $A_{o p t}$ is the optical area of the device. Evaluating $R_{p h}$ for single-nanowire photodetectors is not evident due to the difficulty in quantifying $A_{o p t}$. As the nanowire dimensions are comparable to or smaller than the light wavelength, the optical area is a function of the wavelength, the nanowire shape and the involved refractive indices, and it can be several times larger than the cross-section of the wire exposed to the light. ${ }^{48}$ To be able to compare our results with previous literature, we calculate $R_{p h}$ by considering only the optical power impinging on the nanowire surface. For a linear photodiode, $R_{p h}$ remains constant at a given bias, e.g. sample NW1 has a responsivity $R_{p h}$ of $355 \mathrm{~A} / \mathrm{W}$ at room temperature and under $-3 \mathrm{~V}$ bias. In contrast, in the sublinear regime, $R_{p h}$ depends on the incident power (e.g. in sample NW1 at zero bias, $R_{p h}$ increases from 0.05 
to $0.1 \mathrm{~A} / \mathrm{W}$ when decreasing the impinging power density from $10^{-3}$ to $10^{-2} \mathrm{~W} / \mathrm{cm}^{2}$ ). The variation of the responsivity as a function of bias for NW1 and NW2 is included as table S1 in the Supporting Information.

Next, we study the spectral response of the nanowire photodetector. As the spectral intensity of the Xe lamp used as the excitation source modulates the created photocurrent, it is essential to correct the recorded data, $I_{p h}(\lambda)$, taking into account its power dependence $I_{p h} \sim P^{\beta} .^{49}$ The corrected spectral response at zero bias is presented in figure 3(a) for samples NW1 and NW2, and measurements at $\pm 3 \mathrm{~V}$ are shown in figures 3(c) and (d) for samples NW1 and NW2, respectively. We observe that $I_{p h}$ exhibits a sharp cutoff around $\lambda \approx 363 \mathrm{~nm}$, which corresponds to the $\mathrm{GaN}$ bandgap energy at room temperature. A relatively flat plateau is reported for $\lambda<360 \mathrm{~nm}$, whereas the signal is below the detection threshold of the system for $\lambda>380 \mathrm{~nm}$. These spectral profiles validate that $I_{p h}$ originates in the GaN nanowires themselves, without any detectable contribution of the silicon carrier wafer or potential defects, attesting the high crystalline quality of these nanowires. The drastic drop of absorbance for $\lambda>380 \mathrm{~nm}$ is due to the absence of energy levels in the bandgap. ${ }^{47,50}$ The resulting large difference of the photoresponse between the ultraviolet $(<400 \mathrm{~nm})$ and visible $(>400 \mathrm{~nm})$ ranges makes these devices suitable for selective ultraviolet detection, independently of the applied bias

Interestingly, the cutoff slope of sample NW1 (with low leakage current) is more pronounced under reverse bias than under positive bias (see figure 3 (c) for $\pm 3 \mathrm{~V}$, and a similar trend is observed in the whole $\pm 5 \mathrm{~V}$ range presented as figure $\mathrm{S} 3$ of the Supporting Information). The steepness of the slope is directly linked to the presence of states in the bandgap. In a direct bandgap material, the decay of the response for photon energies below the band gap can be approximated by an exponential expression, following Urbach's rule. ${ }^{51}$ At those wavelengths, the photocurrent is assigned to absorption to/from an exponential tail of states that enters the band gap, with a distribution of states that is characterized by the Urbach energy:

$$
E_{U r b}=\left[\frac{d(\ln \alpha)}{d(h v)}\right]^{-1}
$$

where $\alpha$ is the optical absorption, which we will assume here to be proportional to the photocurrent, and $h v$ is the photon energy. The Urbach energy for undoped GaN planar layers is reported to be around $15 \mathrm{meV}$ in absence of external bias, and increases linearly when an electric field $(E)$ is applied, with $\Delta E_{U r b}[\mathrm{meV}]=1.8 \times 10^{-4} E[\mathrm{~V} / \mathrm{cm}] .52$ This smoothening of 
the absorption cutoff is due to the Franz-Keldysh effect, i.e. it results from the wavefunctions extending into the band gap in the presence of an electric field.

Figure 3(b) shows the variation of $E_{U r b}$ as a function of bias for samples NW1 and NW2. For $\mathrm{V}_{\mathrm{B}}<0$, the value of $E_{U r b}$ remains around $15 \mathrm{meV}$ for $\mathrm{NW} 1$, as expected in bulk undoped $\mathrm{GaN}$, and is slightly higher (20-24 meV) for NW2. In contrast, $E_{U r b}$ increases beyond $30 \mathrm{meV}$ for both samples under forward bias. This evolution of the Urbach's tail can be explained by surface band bending. Under negative bias, the depletion region associated to the junction is larger, reducing the surface band bending and hence producing a more abrupt threshold. Under positive bias, the depletion region is reduced and the response becomes sensitive to the surface band bending. Following ref. ${ }^{53}, E_{U r b} \approx 30 \mathrm{meV}$ could indicate an electric field of $\approx 100 \mathrm{kV} / \mathrm{cm}$, which is in the range of the expected radial electric field due to the surface band bending in the n-region (assuming the Fermi level pinned $0.6 \mathrm{meV}$ below the conduction band, a nanowire diameter in the range of $50-200 \mathrm{~nm}$, and a doping level $N_{D}=5 \times 10^{19} \mathrm{~cm}^{-3}$, the average radial electric field induced by the surface band bending is in the range of 55$150 \mathrm{kV} / \mathrm{cm}$, as shown in figure S4 of the Supporting Information). Alternatively, zero-bias measurements led to high Urbach energies of $51 \mathrm{meV}$ and $46 \mathrm{meV}$ for NW1 and NW2, respectively. Therefore, $E_{U r b}$ is inversely correlated with $\beta$. This trend is consistent with the association of the nonlinearity with surface effects. Thus, a reduction of the surface band bending due to the axial depletion of the nanowire results in sharper spectral cutoff and enhanced linearity.

In planar photodetectors, sublinearity is often associated to persistent photoconductivity. ${ }^{46}$ Therefore, we study the photoresponse dynamics of the nanowires to address this point. The photocurrent decay of sample NW1 after excitation with an ultraviolet pulsed laser (1 ns pulses) is shown as a semi-logarithmic plot in figure 4(a). The time response is on the order of microseconds, i.e. orders of magnitude faster than previous reports involving $\mathrm{GaN}$ nanowire photodetectors containing p-n junctions..$^{24,28}$ These measurements are in fact limited by the resistancexcapacitance (RC) time constant of the setup, as verified by comparing measurements with different values of load resistance (see figure S5 of the Supporting Information). Therefore, the intrinsic response time of the photodetectors under reverse bias is in fact shorter than the measured decay times.

In contrast, sublinear samples are not fast enough to follow the pulsed laser. Therefore, the photocurrent decay of NW2 was analyzed by exciting the nanowire with the same HeCd laser 
used during the linearity studies, chopped at a frequency of $2 \mathrm{~Hz}$. The photocurrent decays in this case were in the order of milliseconds, 3 orders of magnitude slower than for NW1, as it can be observed in figure 4(b).

The decay times for both nanowires, calculated as the time that it takes for the photocurrent to drop from $90 \%$ to $10 \%$ of its maximum value, are represented in figure 4(c). Under reverse bias, the decay time decreases compared to forward bias, and in the case of NW1 the photocurrent decays are almost exponential (see figure 4(a)). However, forward bias makes the photodetection slower and strongly non-exponential, as reported in ref. ${ }^{24}$. These results confirm that there is a correlation between the linearity of the nanowires and their time response. Deviations from linearity are associated to slow, non-exponential photocurrent decays.

Finally, in order to gain a deeper insight into the origin of the photocurrent in the nanowires, EBIC measurements were performed. Scans were carried out starting on the p-contact and moving along the red arrows indicated in the SEM images in figures 1(b-c), with the results represented in figure 5. For nanowire NW1 at zero-bias (see figure 5(a)) a plateau can be observed in the p-doped side, extending around $200 \mathrm{~nm}$ from the p-contact, followed by a decay to zero current. This indicates that carriers generated in the Mg-doped area and in the vicinity of the junction are effectively collected because of the internal electric field of the junction. When negative bias is applied $(-0.3 \mathrm{~V}$ in figure $5(\mathrm{a}))$, the junction is reverse biased and the EBIC current increases several orders of magnitude (similar to the photocurrent in figure 2(a)), and the length of the nanowire reacting to the electron beam extends up to $400 \mathrm{~nm}$ from the pcontact. In contrast, under positive bias $(+0.3 \mathrm{~V}$ in figure $5(\mathrm{a}))$, the current is generated in the whole nanowire length, as expected from the photoconductor-like behavior described above. In the case of NW2, at zero bias, no efficient collection of generated carriers in the vicinity of the p-contact is observed, indicating degraded electrical properties of the p-n junction. Moreover, the EBIC signal under bias is rather stable along the wire axis both for positive and negative bias, which is again consistent with the photoconductive behavior of this nanowire.

\section{DISCUSSION}

Bringing together all the experimental results, low-leakage single axial junction nanowires present a behavior similar to planar junctions when forward or reverse biased. Under reverse bias, the devices are characterized by their linearity, high spectral selectivity and high speed, with almost exponential photocurrent decays, limited by the RC product of the system. Increasing reverse bias leads to enhanced responsivity, due to the broadening of the depletion 
region associated to the junction. Under forward bias, the response scales sublinearly with the optical power. This is explained by the fact that the role of the series resistance of the diode becomes dominant over the junction, whose ohmic value depends on the Fermi level pinning at the nanowire sidewalls. Illumination results in nonlinear changes of the band bending induced by surface states, and hence in a nonlinear photoresponse. This interpretation is supported by a slight degradation of the spectral cutoff of the photodiodes when operated in forward bias, which is explained by a Franz-Keldysh effect due to the electric field originated by the surface band bending. Note that this smoothening of the spectral cutoff is completely different from the dramatically degraded spectral response observed in GaN planar photoconductors. ${ }^{46}$ In this latter case, the sublinearity and slow response are associated to band bending at structural defects (dislocations, grain boundaries) which are known to introduce levels within the semiconductor bandgap. On the contrary, in nanowire photodetectors, the band bending is originating at the m-plane nanowire sidewalls, which are characterized by the absence of energy levels within the bandgap..$^{50}$

An additional feature of the forward-bias performance is that the photocurrent decays become slower and non-exponential, contrary to reverse bias. This is again associated to the surface band bending, which radially separates electrons and holes, one of the charge carrier species traveling through the nanowire core and the other one attracted by the nanowire sidewalls. When the excitation source is switched off, the recombination of excess carriers has to overcome the potential barrier associated to the surface band bending, which leads to a long and non-exponential transient response..$^{54}$

In the case of junctions with significant leakage current, their behavior can be modeled as a diode with a shunt resistance, whose response to illumination can be dominant over the junction itself. This results in a sublinear behavior of the photocurrent, even under reverse bias, and slow (millisecond) and non-exponential photocurrent response to pulsed excitation.

A particularity of these GaN nanowires is their behavior observed at zero bias. At zero bias, the photocurrent measured in single-nanowire junctions is strongly sublinear in all the cases, as it was previously observed for GaN-based single-nanowire photodetectors where the internal electric field was generated by the polarization difference in a heterostructure. ${ }^{15,18}$ This behavior comes with a drastic drop of the responsivity and a slight degradation of the spectral cutoff, which could also be attributed to a Franz-Keldysh effect. This performance is in contrast to the linearity of photovoltaic devices based on planar layers. ${ }^{46}$ Identifying the origin of this deviation will be very important to improve the efficiency of nanowire photovoltaic detectors. However, 
with the data currently available, it is difficult to discern if the origin of these phenomena is due to a higher sensitivity to surface states at zero bias or to the potential barrier induced by nonideal ohmic contacts.

Comparing the experimental results with previous literature, this paper demonstrates the first GaN-based nanowire photodetector that combines simultaneously linearity, spectral contrast and speed (microsecond scale). It demonstrates that it is possible to fabricate axial p-n photodiodes in a wire with the electric field induced by the $\mathrm{p}-\mathrm{n}$ junction being dominant over surface effects. Let us remind here that this was not the case in devices with an axial electric field induced by a difference in polarization..$^{15}$ In this latter case, the phocurrent recovery time remained in the millisecond range even in linear (fully depleted) devices. ${ }^{18}$

In conclusion, we presented a study of the performance of a single $\mathrm{GaN}$ axial $\mathrm{p}$-n junction nanowire photodetector. The design of the device incorporates a $\mathrm{p}^{+} / \mathrm{n}^{+}$tunnel junction to facilitate the electrical access to the $\mathrm{p}$ region, enabling the use of the same metal scheme for both ends of the wire. Under reverse bias, the contacted wires are characterized by their linearity, high spectral selectivity and high speed of microsecond time response (limited by the $\mathrm{RC}$ product of the system). Under forward bias, the response to ultraviolet radiation is higher, but it scales sublinearly with the optical power. This is explained by the role of the series resistance of the diode becoming dominant over the junction, whose ohmic value depends on the Fermi level pinning at the nanowire sidewalls. This sublinearity comes with a slight degradation of the spectral cutoff, explained by a Franz-Keldysh effect due to the electric field originating from the surface band bending, and a degradation of the time response, with photocurrent decays becoming significantly slower and non-exponential. At zero bias, the photocurrent measured in single-nanowire junctions is strongly sublinear in all the cases. Overall, understanding the physical effects of the photodetection in a single nanowire is crucial to further optimize p-n junction nanowire photodetectors and nanowire solar cells.

\section{SUPPORTING INFORMATION}

The Supporting Information is available free of charge on the ACS Publications website at DOI:10.1021/acs.nanolett.9b02040

Measurements of the surface potential of NW2 using KPFM, complementary results confirming 
the linearity under reverse bias for another single nanowire, values for the responsivity at different bias and illumination conditions, detailed variation of the spectral response of NW1 as a function of bias, calculations of the radial internal electric field induced by surface states, and variation of the time response of NW1 as a function of the load resistance (PDF)

\section{AUTHOR INFORMATION}

Corresponding Author

*E-mail: eva.monroy@cea.fr.

ORCID

S. Cuesta: 0000-0003-0262-5875

M. Spies: 0000-0002-3570-3422

V. Boureau: 0000-0001-6251-5892

F. Donatini: 0000-0001-6556-1683

M. Hocevar: 0000-0001-5949-5480

M. I. den Hertog: 0000-0003-0781-9249

E. Monroy: 0000-0001-5481-3267

Notes

The authors declare no competing financial interest.

\section{ACKNOWLEDGEMENTS}

This work is supported by the French National Research Agency via the UVLASE program (ANR-18-CE24-0014), and by the Auvergne-Rhône-Alpes region (grant PEAPLE). This project has also received funding from the European Research Council under the European Union's H2020 Research and Innovation programme for the e-See project (grant \#758385). We benefited from access to the Nano-characterization platform (PFNC) in CEA Minatec and to the Nano-fab cleanroom facilities from CNRS-Institute Néel, Grenoble. Thanks are due to Bruno Fernandez and Jean-François Motte for their technical support. We also acknowledge PAMBE support from Y. Curé and Y. Genuist.

\section{REFERENCES}

(1) Otnes, G.; Borgström, M. T. Towards High Efficiency Nanowire Solar Cells. Nano Today 2017, 12, 31-45. https://doi.org/10.1016/j.nantod.2016.10.007.

(2) Jia, G.; Steglich, M.; Sill, I.; Falk, F. Core-Shell Heterojunction Solar Cells on Silicon Nanowire Arrays. Sol. Energy Mater. Sol. Cells 2012, 96, 226-230. https://doi.org/10.1016/j.solmat.2011.09.062.

(3) Haverkort, J. E. M.; Garnett, E. C.; Bakkers, E. P. A. M. Fundamentals of the Nanowire Solar Cell: Optimization of the Open Circuit Voltage. Appl. Phys. Rev. 2018, 5 (3), 031106. https://doi.org/10.1063/1.5028049.

(4) Xu, Y.; Gong, T.; Munday, J. N. The Generalized Shockley-Queisser Limit for Nanostructured Solar Cells. Sci. Rep. 2015, 5, 13536. 
(5) Krogstrup, P.; Jørgensen, H. I.; Heiss, M.; Demichel, O.; Holm, J. V.; Aagesen, M.; Nygard, J.; Fontcuberta i Morral, A. Single-Nanowire Solar Cells beyond the ShockleyQueisser Limit. Nat. Photonics 2013, 7 (4), 306-310. https://doi.org/10.1038/nphoton.2013.32.

(6) Lähnemann, J.; Browne, D. A.; Ajay, A.; Jeannin, M.; Vasanelli, A.; Thomassin, J.-L.; Bellet-Amalric, E.; Monroy, E. Near- and Mid-Infrared Intersubband Absorption in Topdown GaN/AlN Nano- and Micro-Pillars. Nanotechnology 2019, 30 (5), 054002. https://doi.org/10.1088/1361-6528/aaef72.

(7) Lou, Z.; Shen, G. Flexible Photodetectors Based on 1D Inorganic Nanostructures. $A d v$. Sci. 2016, 3 (6), 1500287. https://doi.org/10.1002/advs.201500287.

(8) Zhang, H.; Dai, X.; Guan, N.; Messanvi, A.; Neplokh, V.; Piazza, V.; Vallo, M.; Bougerol, C.; Julien, F. H.; Babichev, A.; et al. Flexible Photodiodes Based on Nitride Core/Shell p-n Junction Nanowires. ACS Appl. Mater. Interfaces 2016, 8 (39), 26198-26206. https://doi.org/10.1021/acsami.6b06414.

(9) Vj, L.; Oh, J.; Nayak, A. P.; Katzenmeyer, A. M.; Gilchrist, K. H.; Grego, S.; Kobayashi, N. P.; Wang, S.-Y.; Talin, A. A.; Dhar, N. K.; et al. A Perspective on Nanowire Photodetectors: Current Status, Future Challenges, and Opportunities. IEEE J. Sel. Top. Quantum Electron. 2011, $17 \quad$ (4), 1002-1032. https://doi.org/10.1109/JSTQE.2010.2093508.

(10) Soci, C.; Zhang, A.; Bao, X.-Y.; Kim, H.; Lo, Y.; Wang, D. Nanowire Photodetectors. J. Nanosci. Nanotechnol. 2010, 10 (3), 1430-1449. https://doi.org/10.1166/jnn.2010.2157.

(11) Spies, M.; Monroy, E. Nanowire Photodetectors Based on Wurtzite Semiconductor Heterostructures. Semicond. Sci. Technol. 2019, 34 (5), 053002. https://doi.org/10.1088/1361-6641/ab0cb8.

(12) Cui, Y.; Wang, J.; Plissard, S. R.; Cavalli, A.; Vu, T. T. T.; van Veldhoven, R. P. J.; Gao, L.; Trainor, M.; Verheijen, M. A.; Haverkort, J. E. M.; et al. Efficiency Enhancement of InP Nanowire Solar Cells by Surface Cleaning. Nano Lett. 2013, 13 (9), 4113-4117. https://doi.org/10.1021/nl4016182.

(13) Wallentin, J.; Anttu, N.; Asoli, D.; Huffman, M.; Aberg, I.; Magnusson, M. H.; Siefer, G.; Fuss-Kailuweit, P.; Dimroth, F.; Witzigmann, B.; et al. InP Nanowire Array Solar Cells Achieving 13.8\% Efficiency by Exceeding the Ray Optics Limit. Science 2013, 339(6123), 1057-1060. https://doi.org/10.1126/science.1230969.

(14) Aberg, I.; Vescovi, G.; Asoli, D.; Naseem, U.; Gilboy, J. P.; Sundvall, C.; Dahlgren, A.; Svensson, K. E.; Anttu, N.; Bjork, M. T.; et al. A GaAs Nanowire Array Solar Cell With 15.3\% Efficiency at 1 Sun. IEEE J. Photovolt. 2016, 6 (1), 185-190. https://doi.org/10.1109/JPHOTOV.2015.2484967.

(15) Spies, M.; den Hertog, M. I.; Hille, P.; Schörmann, J.; Polaczyński, J.; Gayral, B.; Eickhoff, M.; Monroy, E.; Lähnemann, J. Bias-Controlled Spectral Response in GaN/AlN Single-Nanowire Ultraviolet Photodetectors. Nano Lett. 2017, 17 (7), 4231-4239. https://doi.org/10.1021/acs.nanolett.7b01118.

(16) Calarco, R.; Marso, M.; Richter, T.; Aykanat, A. I.; Meijers, R.; v.d. Hart, A.; Stoica, T.; Lüth, H. Size-Dependent Photoconductivity in MBE-Grown GaN-Nanowires. Nano Lett. 2005, 5 (5), 981-984. https://doi.org/10.1021/nl0500306.

(17) Sanford, N. A.; Blanchard, P. T.; Bertness, K. A.; Mansfield, L.; Schlager, J. B.; Sanders, A. W.; Roshko, A.; Burton, B. B.; George, S. M. Steady-State and Transient Photoconductivity in c-Axis GaN Nanowires Grown by Nitrogen-Plasma-Assisted Molecular Beam Epitaxy. J. Appl. Phys. 2010, 107 (3), 034318. https://doi.org/10.1063/1.3275888.

(18) Spies, M.; Polaczyński, J.; Ajay, A.; Kalita, D.; Luong, M. A.; Lähnemann, J.; Gayral, B.; den Hertog, M. I.; Monroy, E. Effect of the Nanowire Diameter on the Linearity of the Response of GaN-Based Heterostructured Nanowire Photodetectors. Nanotechnology 2018, 29 (25), 255204. https://doi.org/10.1088/1361-6528/aab838.

(19) Tian, B.; Zheng, X.; Kempa, T. J.; Fang, Y.; Yu, N.; Yu, G.; Huang, J.; Lieber, C. M. Coaxial Silicon Nanowires as Solar Cells and Nanoelectronic Power Sources. Nature 2007, 449 (7164), 885-889. https://doi.org/10.1038/nature06181. 
(20) Czaban, J. A.; Thompson, D. A.; LaPierre, R. R. GaAs Core-Shell Nanowires for Photovoltaic Applications. Nano Lett. 2009, 9 (1), 148-154. https://doi.org/10.1021/nl802700u.

(21) Colombo, C.; Heiß, M.; Grätzel, M.; Fontcuberta i Morral, A. Gallium Arsenide P-i-n Radial Structures for Photovoltaic Applications. Appl. Phys. Lett. 2009, 94 (17), 173108. https://doi.org/10.1063/1.3125435.

(22) Holm, J. V.; Jørgensen, H. I.; Krogstrup, P.; Nygård, J.; Liu, H.; Aagesen, M. SurfacePassivated GaAsP Single-Nanowire Solar Cells Exceeding 10\% Efficiency Grown on Silicon. Nat. Commun. 2013, 4(1). https://doi.org/10.1038/ncomms2510.

(23) Tchernycheva, M.; Rigutti, L.; Jacopin, G.; de Luna Bugallo, A.; Lavenus, P.; Julien, F. H.; Timofeeva, M.; Bouravleuv, A. D.; Cirlin, G. E.; Dhaka, V.; et al. Photovoltaic Properties of GaAsP Core-Shell Nanowires on Si(001) Substrate. Nanotechnology 2012, 23 (26), 265402. https://doi.org/10.1088/0957-4484/23/26/265402.

(24) Zhang, H.; Guan, N.; Piazza, V.; Kapoor, A.; Bougerol, C.; Julien, F. H.; Babichev, A. V.; Cavassilas, N.; Bescond, M.; Michelini, F.; et al. Comprehensive Analyses of Core-Shell InGaN/GaN Single Nanowire Photodiodes. J. Phys. Appl. Phys. 2017, 50 (48), 484001. https://doi.org/10.1088/1361-6463/aa935d.

(25) Kempa, T. J.; Tian, B.; Kim, D. R.; Hu, J.; Zheng, X.; Lieber, C. M. Single and Tandem Axial $p-i-n$ Nanowire Photovoltaic Devices. Nano Lett. 2008, 8 (10), 3456-3460. https://doi.org/10.1021/nl8023438.

(26) Lysov, A.; Vinaji, S.; Offer, M.; Gutsche, C.; Regolin, I.; Mertin, W.; Geller, M.; Prost, W.; Bacher, G.; Tegude, F.-J. Spatially Resolved Photoelectric Performance of Axial GaAs Nanowire Pn-Diodes. Nano Res. 2011, 4 (10), 987-995. https://doi.org/10.1007/s12274011-0155-4.

(27) Son, M. S.; Im, S. I.; Park, Y. S.; Park, C. M.; Kang, T. W.; Yoo, K.-H. Ultraviolet Photodetector Based on Single GaN Nanorod p-n Junctions. Mater. Sci. Eng. C2006, 26 (5-7), 886-888. https://doi.org/10.1016/j.msec.2005.09.089.

(28) de Luna Bugallo, A.; Tchernycheva, M.; Jacopin, G.; Rigutti, L.; Julien, F. H.; Chou, S.T.; Lin, Y.-T.; Tseng, P.-H.; Tu, L.-W. Visible-Blind Photodetector Based on p-i-n Junction GaN Nanowire Ensembles. Nanotechnology 2010, 21 (31), 315201. https://doi.org/10.1088/0957-4484/21/31/315201.

(29) Jacopin, G.; De Luna Bugallo, A.; Rigutti, L.; Lavenus, P.; Julien, F. H.; Lin, Y.-T.; Tu, L.-W.; Tchernycheva, M. Interplay of the Photovoltaic and Photoconductive Operation Modes in Visible-Blind Photodetectors Based on Axial p-i-n Junction GaN Nanowires. Appl. Phys. Lett. 2014, 104 (2), 023116. https://doi.org/10.1063/1.4860968.

(30) Bulgarini, G.; Reimer, M. E.; Hocevar, M.; Bakkers, E. P. A. M.; Kouwenhoven, L. P.; Zwiller, V. Avalanche Amplification of a Single Exciton in a Semiconductor Nanowire. Nat. Photonics 2012, 6 (7), 455-458. https://doi.org/10.1038/nphoton.2012.110.

(31) Jain, V.; Heurlin, M.; Barrigon, E.; Bosco, L.; Nowzari, A.; Shroff, S.; Boix, V.; Karimi, M.; Jam, R. J.; Berg, A.; et al. InP/InAsP Nanowire-Based Spatially Separate Absorption and Multiplication Avalanche Photodetectors. ACS Photonics 2017, 4 (11), 2693-2698. https://doi.org/10.1021/acsphotonics.7b00389.

(32) Senanayake, P.; Hung, C.-H.; Farrell, A.; Ramirez, D. A.; Shapiro, J.; Li, C.-K.; Wu, Y.R.; Hayat, M. M.; Huffaker, D. L. Thin 3D Multiplication Regions in Plasmonically Enhanced Nanopillar Avalanche Detectors. Nano Lett. 2012, 12 (12), 6448-6452. https://doi.org/10.1021/nl303837y.

(33) Chuang, L. C.; Sedgwick, F. G.; Chen, R.; Ko, W. S.; Moewe, M.; Ng, K. W.; Tran, T.-T. D.; Chang-Hasnain, C. GaAs-Based Nanoneedle Light Emitting Diode and Avalanche Photodiode Monolithically Integrated on a Silicon Substrate. Nano Lett. 2011, 11 (2), 385-390. https://doi.org/10.1021/nl102988w.

(34) Farrell, A. C.; Meng, X.; Ren, D.; Kim, H.; Senanayake, P.; Hsieh, N. Y.; Rong, Z.; Chang, T.-Y.; Azizur-Rahman, K. M.; Huffaker, D. L. InGaAs-GaAs Nanowire Avalanche Photodiodes Toward Single-Photon Detection in Free-Running Mode. Nano Lett. 2019, 19 (1), 582-590. https://doi.org/10.1021/acs.nanolett.8b04643.

(35) Kayes, B. M.; Atwater, H. A.; Lewis, N. S. Comparison of the Device Physics Principles 
of Planar and Radial P-n Junction Nanorod Solar Cells. J. Appl. Phys. 2005, 97 (11), 114302. https://doi.org/10.1063/1.1901835.

(36) Yoshizawa, M.; Kikuchi, A.; Mori, M.; Fujita, N.; Kishino, K. Growth of Self-Organized GaN Nanostructures on Al2O3(0001) by RF-Radical Source Molecular Beam Epitaxy. Jpn. J. Appl. Phys. 1997, 36 (4B), L459. https://doi.org/10.1143/JJAP.36.L459.

(37) Sanchez-Garcia, M. A.; Calleja, E.; Monroy, E.; Sanchez, F. J.; Calle, F.; Muñoz, E.; Beresford, R. The Effect of the III/V Ratio and Substrate Temperature on the Morphology and Properties of GaN- and AlN-Layers Grown by Molecular Beam Epitaxy on $\mathrm{Si}(1 \mathrm{1}$ 1). J. Cryst. Growth 1998, 183 (1-2), 23-30. https://doi.org/10.1016/S00220248(97)00386-2.

(38) den Hertog, M. I.; González-Posada, F.; Songmuang, R.; Rouviere, J. L.; Fournier, T.; Fernandez, B.; Monroy, E. Correlation of Polarity and Crystal Structure with Optoelectronic and Transport Properties of GaN/AlN/GaN Nanowire Sensors. Nano Lett. 2012, 12 (11), 5691-5696. https://doi.org/10.1021/nl302890f.

(39) Ajay, A.; Lim, C. B.; Browne, D. A.; Polaczynski, J.; Bellet-Amalric, E.; den Hertog, M. I.; Monroy, E. Intersubband Absorption in $\mathrm{Si}^{-}$and Ge-Doped GaN/AlN Heterostructures in Self-Assembled Nanowire and 2D Layers. Phys. Status Solidi B 2017, 254 (8), 1600734. https://doi.org/10.1002/pssb.201600734.

(40) Birner, S.; Zibold, T.; Andlauer, T.; Kubis, T.; Sabathil, M.; Trellakis, A.; Vogl, P. Nextnano: General Purpose 3-D Simulations. IEEE Trans. Electron Devices 2007, 54 (9), 2137-2142. https://doi.org/10.1109/TED.2007.902871.

(41) Seong-Ran Jeon; Myong Soo Cho; Min-A Yu; Gye Mo Yang. GaN-Based Light-Emitting Diodes Using Tunnel Junctions. IEEE J. Sel. Top. Quantum Electron. 2002, 8(4), 739743. https://doi.org/10.1109/JSTQE.2002.800847.

(42) Young, E. C.; Yonkee, B. P.; Wu, F.; Oh, S. H.; DenBaars, S. P.; Nakamura, S.; Speck, J. S. Hybrid Tunnel Junction Contacts to III-Nitride Light-Emitting Diodes. Appl. Phys. Express 2016, $9(2)$, 022102. https://doi.org/10.7567/APEX.9.022102.

(43) Sarwar, A. T. M. G.; May, B. J.; Deitz, J. I.; Grassman, T. J.; McComb, D. W.; Myers, R. C. Tunnel Junction Enhanced Nanowire Ultraviolet Light Emitting Diodes. Appl. Phys. Lett. 2015, 107(10), 101103. https://doi.org/10.1063/1.4930593.

(44) Minj, A.; Cros, A.; Auzelle, T.; Pernot, J.; Daudin, B. Direct Assessment of p-n Junctions in Single GaN Nanowires by Kelvin Probe Force Microscopy. Nanotechnology 2016, 27 (38), 385202. https://doi.org/10.1088/0957-4484/27/38/385202.

(45) Sun, X.; Wang, X.; Wang, P.; Sheng, B.; Li, M.; Su, J.; Zhang, J.; Liu, F.; Rong, X.; Xu, F.; et al. Identifying a Doping Type of Semiconductor Nanowires by Photoassisted Kelvin Probe Force Microscopy as Exemplified for GaN Nanowires. Opt. Mater. Express 2017, 7(3), 904. https://doi.org/10.1364/OME.7.000904.

(46) Monroy, E.; Omnès, F.; Calle, F. Wide-Bandgap Semiconductor Ultraviolet Photodetectors. Semicond. Sci. Technol. 2003, 18 (4), R33-R51. https://doi.org/10.1088/0268-1242/18/4/201.

(47) González-Posada, F.; Songmuang, R.; Den Hertog, M.; Monroy, E. Room-Temperature Photodetection Dynamics of Single GaN Nanowires. Nano Lett. 2012, 12 (1), 172-176. https://doi.org/10.1021/nl2032684.

(48) Heiss, M.; Russo-Averchi, E.; Dalmau-Mallorquí, A.; Tütüncüoğlu, G.; Matteini, F.; Rüffer, D.; Conesa-Boj, S.; Demichel, O.; Alarcon-Lladó, E.; Fontcuberta i Morral, A. IIIV Nanowire Arrays: Growth and Light Interaction. Nanotechnology 2014, 25 (1), 014015. https://doi.org/10.1088/0957-4484/25/1/014015.

(49) González-Posada, F.; Songmuang, R.; Hertog, M. D.; Monroy, E. Responsivity and Photocurrent Dynamics in Single GaN Nanowires. Phys. Status Solidi C 2012, 9 (3-4), 642-645. https://doi.org/10.1002/pssc.201100382.

(50) Bertelli, M.; Löptien, P.; Wenderoth, M.; Rizzi, A.; Ulbrich, R.; Righi, M.; Ferretti, A.; Martin-Samos, L.; Bertoni, C.; Catellani, A. Atomic and Electronic Structure of the Nonpolar GaN(1-100) Surface. Phys. Rev. $B$ 2009, 80 (11), 115324. https://doi.org/10.1103/PhysRevB.80.115324.

(51) Urbach, F. The Long-Wavelength Edge of Photographic Sensitivity and of the Electronic 
Absorption of Solids. Phys. Rev. 1953, 92 (5), 1324-1324. https://doi.org/10.1103/PhysRev.92.1324.

(52) Jacobson, M. A.; Konstantinov, O. V.; Nelson, D. K.; Romanovskii, S. O.; Hatzopoulos, Z. Absorption Spectra of GaN: Film Characterization by Urbach Spectral Tail and the Effect of Electric Field. J. Cryst. Growth 2001, 230 (3), 459-461. https://doi.org/10.1016/S0022-0248(01)01246-5.

(53) Jacobson, M. A.; Konstantinov, O. V.; Nelson, D. K.; Romanovskii, S. O.; Hatzopoulos, Z. Absorption Spectra of GaN: Film Characterization by Urbach Spectral Tail and the Effect of Electric Field. J. Cryst. Growth 2001, 230 (3-4), 459-461. https://doi.org/10.1016/S0022-0248(01)01246-5.

(54) Garrido, J. A.; Monroy, E.; Izpura, I.; Muñoz, E. Photoconductive Gain Modelling of GaN Photodetectors. Semicond. Sci. Technol. 1998, 13 (6), 563-568. https://doi.org/10.1088/0268-1242/13/6/005. 

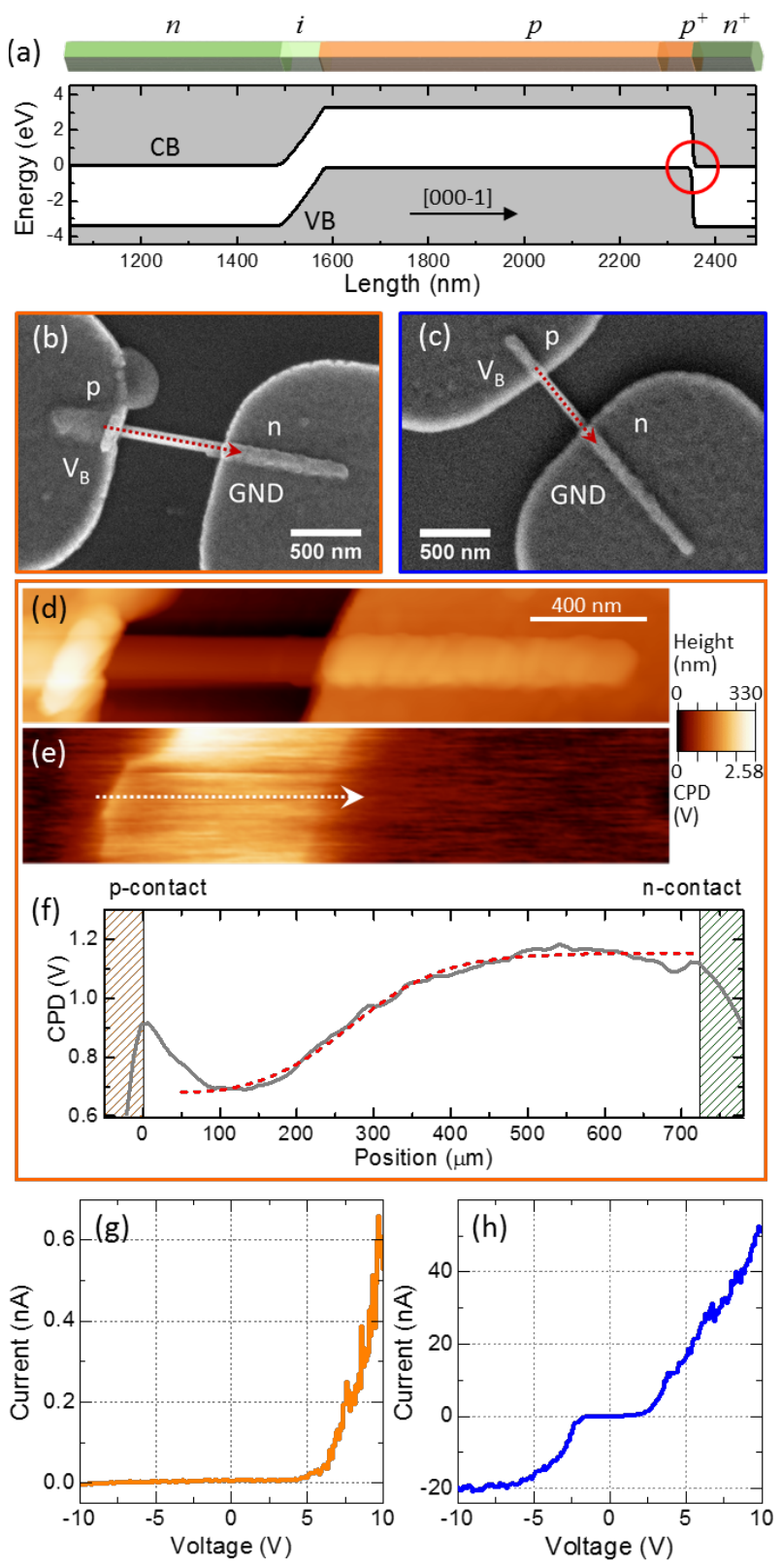

Figure 1. (a) Schematics of the GaN p-n junction nanowires under study and their band diagram calculated with the Nextnano3 software using a one-dimensional approximation. The red circle outlines the $\mathrm{n}^{+} / \mathrm{p}^{+}$tunnel junction that facilitates the electrical contact to the $\mathrm{p}$ region. (b,c) SEM images of the contacted nanowires (b) NW1 and (c) NW2. The voltage convention is indicated for both images, with bias applied to the $\mathrm{GaN}$ cap $\left(\mathrm{V}_{\mathrm{B}}\right)$ whereas the $\mathrm{GaN}$ stem is grounded (GND). (d) Height and (e) CPD extracted from KPFM measurements of NW1. (f) CPD profile along the arrow in (e). The dashed line is a sigmoidal fit. Shadowed orange and green areas mark the location of the $\mathrm{p}$ and $\mathrm{n}$ contacts, respectively. (g,h) I-V characteristics at room temperature of samples (g) NW1 and (h) NW2. 


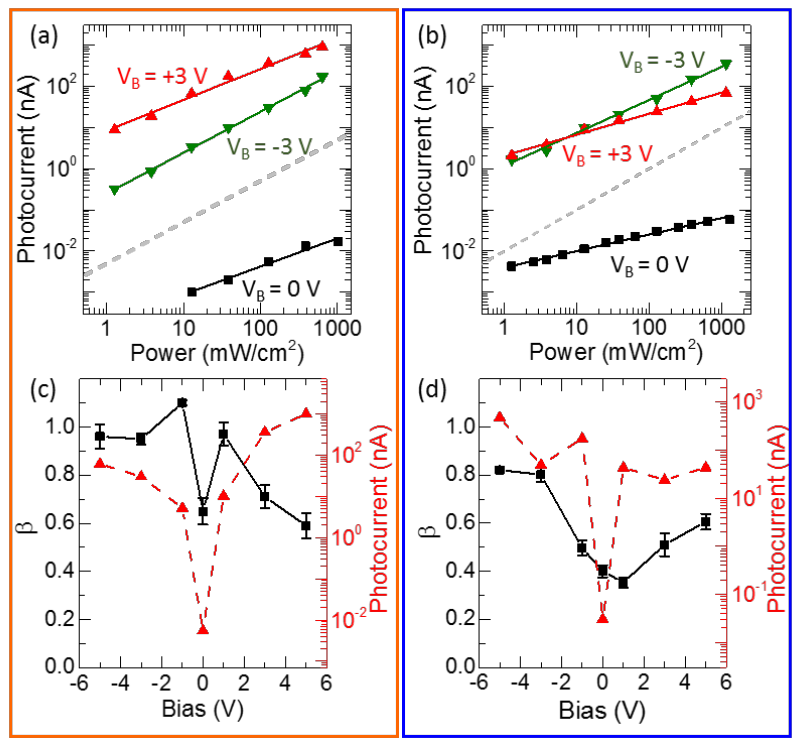

Figure 2. (a-b) Variation of the photocurrent $\left(I_{p h}\right)$ as a function of the impinging optical power $(P)$ at $325 \mathrm{~nm}$ for samples (a) NW1 and (b) NW2, polarized at $-3,0$ and $+3 \mathrm{~V}$ bias. Solid lines are fits to the power law $I_{p h} \sim P^{\beta}$. The gray dashed line illustrates the slope corresponding to $\beta=1$, i.e. photocurrent scaling linearly with the optical power. (c-d) Variation of the fitting parameter $\beta$ and the photocurrent under $0.13 \mathrm{~W} / \mathrm{cm}^{2}$ excitation as a function of bias, for samples (c) NW1 and (d) NW2.
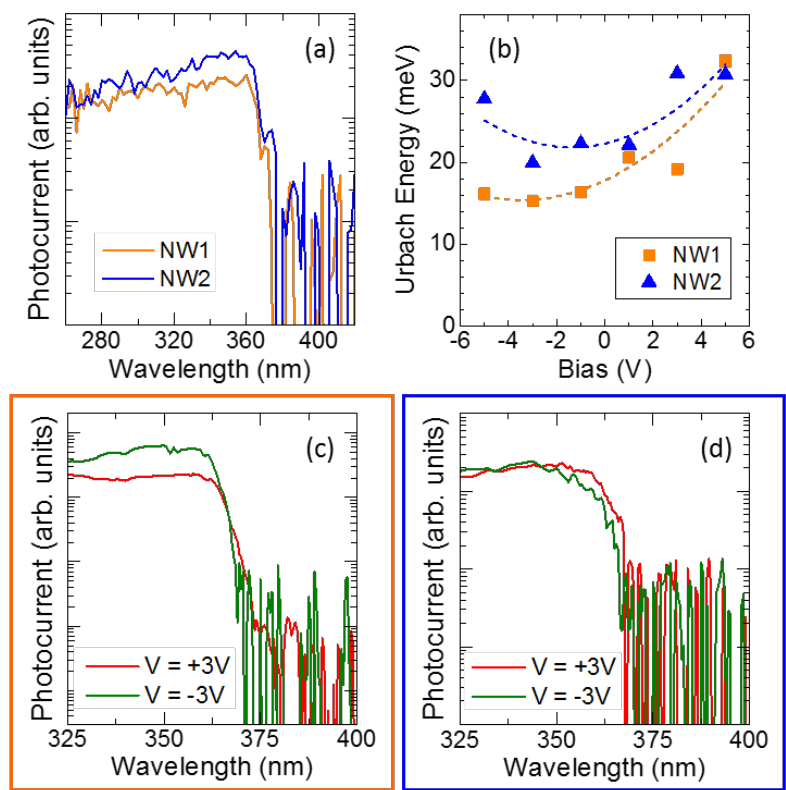

Figure 3. (a) Comparison of the spectral response of NW1 and NW2 at zero-bias. Data are corrected by the Xe lamp emission spectrum taking the sublinearity of the response into account. (b) Urbach energy extracted from spectral photocurrent measurements as a function of bias, for NW1 and NW2. Dashed lines are guides for the eye. (c-d) Spectral response of (c) NW1 and (d) NW2 at +3 V and -3 V bias. Data are corrected by the Xe-lamp emission spectrum taking the linearity or sublinearity of the response into account. 

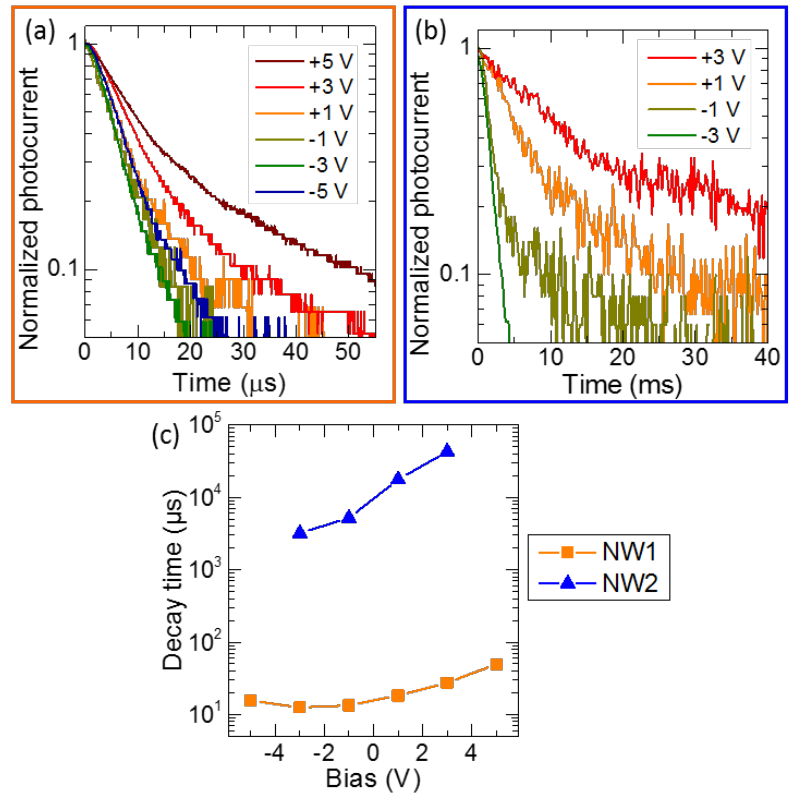

Figure 4. (a) Photocurrent decay from nanowire NW1 after illumination with a pulsed ultraviolet laser $\left(\lambda=266 \mathrm{~nm}, 1 \mathrm{~ns}\right.$ pulses with a frequency of $\left.8 \mathrm{kHz}, 280 \mathrm{~mW} / \mathrm{cm}^{2}\right)$. (b) Photocurrent decay from nanowire NW2 after illumination with a continuous-wave ultraviolet $\left(\lambda=325 \mathrm{~nm}, 1.02 \mathrm{~W} / \mathrm{cm}^{2}\right)$ chopped at $2 \mathrm{~Hz}$. Temporal response of the nanowires measured at different bias between $-5 \mathrm{~V}$ and $+5 \mathrm{~V}$ for samples NW1 (a) and NW2 (b). (c) Variation of decay time as function of bias for samples NW1 and NW2. 

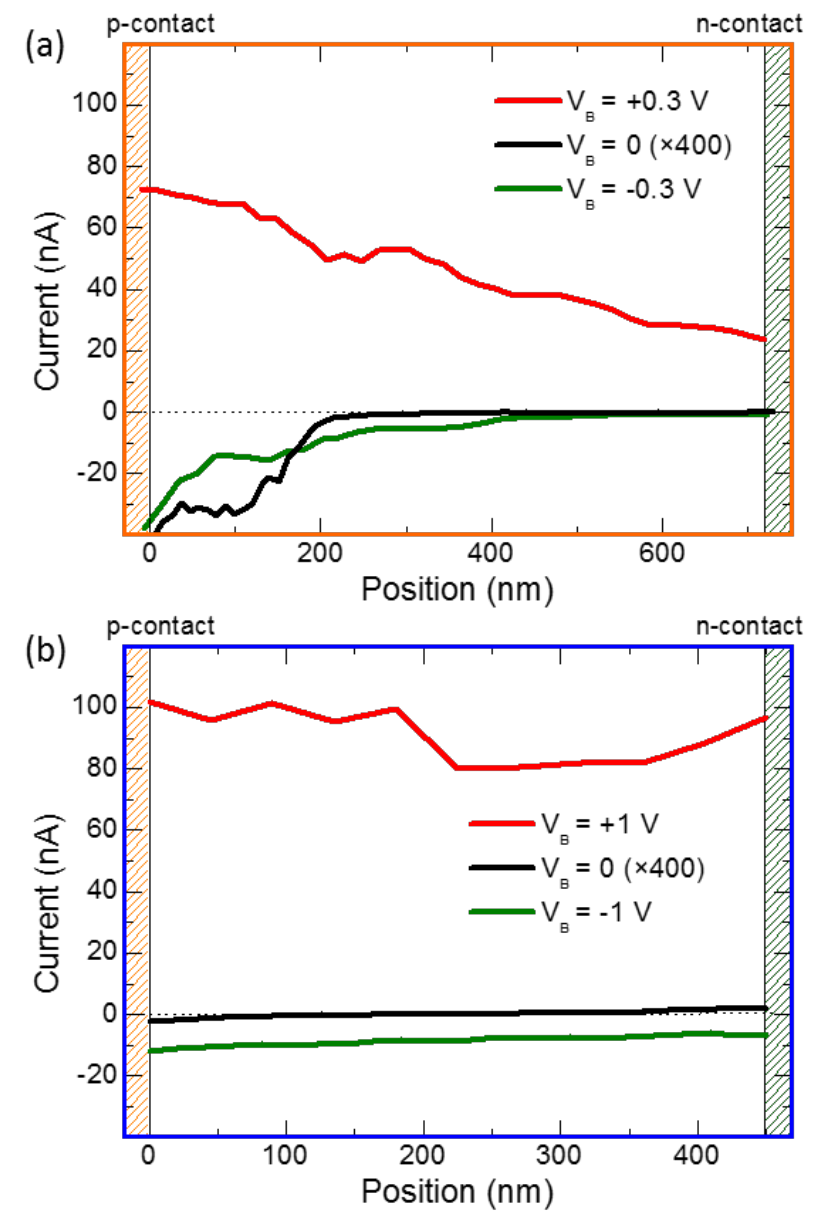

Figure 5. EBIC scan along the nanowires (a) NW1 at $+0.3 \mathrm{~V}, 0 \mathrm{~V}$ and $-0.3 \mathrm{~V}$, and (b) NW2 at $+1 \mathrm{~V}, 0 \mathrm{~V}$ and $-1 \mathrm{~V}$. The scans were performed following the red arrows in figures $1(\mathrm{~b})$ and (c), respectively. Shadowed orange and green areas mark the location of the $p$ and $n$ contacts, respectively. Current values for zero-bias have been multiplied by a factor of 400 for a better legibility. 
TOC Graphic

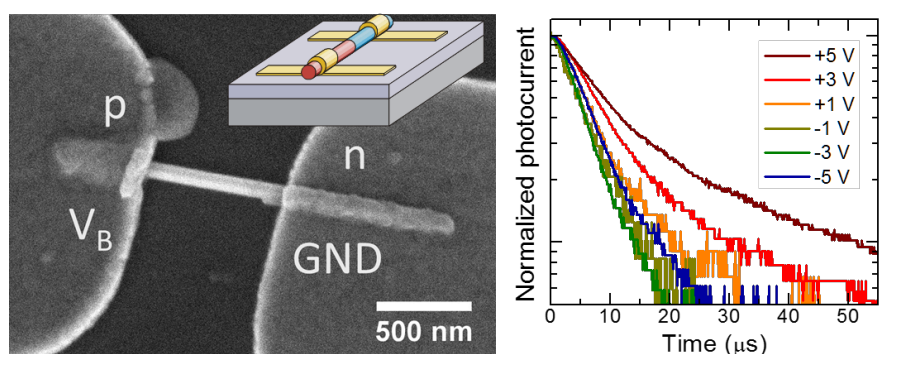

